\section{Detection of changes in spatial position: III. Dot number or dot density?*}

\author{
IRWIN POLLACK† \\ The University of Michigan, Ann Arbor, Michigan 41804
}

Better detection of changes in spatial position is achieved within random dot patterns consisting of a small number of dots rather than a large number of dots. Why? The result might be related directly to the number of display elements which must be monitored and thereby linked to the sharing of attention across display elements. The result, previously obtained for displays of constant area, might be related to the density of display elements and thereby linked to proximity relations within spatial patterns. Or the result may be due to the local separation of neighboring dots when inquiring about the state of a specific dot. The present study attempts to unconfound these factors. It is shown that performance is related to the number of dots that must be monitored, irrespective of dot density; that dot density is effective primarily in the inquiry of the state of specific elements; and then principally as it relates to the separation of the queried element from its neighbors.

At least two classes of sharing-of-attention hypotheses may be distinguished for the extraction of information from visual displays. In one, there is a sharing of attention across display elements. This approach characterizes Rumelhart's (1971) modeling of visual information processing. Processing capability is shared among all of the elements of the display. The larger the number of elements, the smaller will be the processing allocated to each element; and performance should decrease with the number of display elements. Within limits imposed by visual acuity, performance would be independent of the absolute size of the display.

A related hypothesis considers the sharing of attention across the entire display area. In tasks with independently randomly positioned elements, such factors as the average separation among elements would affect the capacity allocated to the display elements. The larger the spacing among elements, the smaller will be the processing allocated to each element. For displays with a fixed number of display elements, within the limits imposed by visual acuity, performance would improve with smaller and smaller displays.

The present paper reexamines the detection of changes in spatial position for random-dot display patterns and for individual dots within such

*This research was supported in part by Grant GB 14036X of the National Science Foundation. The author is indebted to Nancy Mandel, who supervised the experimental terts; to Don Mayer, who developed the PDP-8 programs based upon programs oridinally developed by Lon Radin; and to Robert Shea, who processed the experimental results.

tMailing address: Mental Health Research Institute, University of Michigan, Ann Arbor, Mich. 48104. displays. For a fixed number of displaced elements, detection performance is sensitive to the total number of dots in the random dot pattern. This result obtains whether we inquire whether any dot of the display (Pollack, 1972) or whether a specifically designated dot was, or was not, displaced. 1 The result is sensible in terms of the sharing of attention across display elements and, more importantly, meets the intuitive criterion of common sense. A potentially serious confounding, however, arises in the interpretation of the finding. Since the previous studies were carried out with a fixed display area, we cannot distinguish between the effects of the number of display elements and the effects of display density - the number of display elements per unit area. Whereas the number of display elements is primarily related to the first class of sharing-of-attention hypotheses, display density is primarily related to the second class of sharing-of-attention hypotheses. The present study attempts to unravel the previous confounding by exploring the interaction among number of display elements and display density.

\section{METHOD}

Generation of

Random Dot Patterns

Random dot patterns were were painted upon a Tektronix 602 display scope equipped with a fast P-15 phosphor. Details have previously been described (Pollack, 1972 ${ }^{1}$ ). The variables, which were manipulated under program control, are presented in Table 1.

A field of $\mathbf{n}$ randomly positioned dots were displayed for duration $t$. Following a wait of IFI, a second generated by a PDP-8 computer and display field was presented for duration $t$ with $(n-1)$ dots in the same position as in the first field and with one dot displaced. The displacement was in any one of four randomly chosen directions: NE, SE, $\mathrm{SW}$, or NW, of distance $d$ upon the $\mathrm{x}$ and $y$-coordinates. Note that $d$, and other distances upon the display, are specified in terms of the horizontal or vertical component, not the resultant.

\section{Procedure}

Two test paradigms were examined: detection of any change in the dot pattern and detection of the state of specifically designated dot in the pattern. These task requirements correspond to two related tasks of Sekuler and Abrams (1968).

In tests where $O$ discriminated any change in position, one dot was displaced in one-half of the trials and no dots were displaced in the other half of the trials. O responded whether or not any dot was displaced. There was no requirement to identify the displaced dot. In tests where $O$ detected whether a specifically designated dot had, or had not, been displaced, a query circle of $2.8 \mathrm{~mm}$ diam surrounded one of the dots of the second field after a wait of IQI. In one-half of the trials, the query circle surrounded a displaced dot; in one-half of the trials, the query surrounded a nondisplaced dot; and $O$ responded whether or not the queried dot had been displaced.

It would first appear that the test requirements for the two experimental paradigms are identical for the

Table 1

Manipulated Variables Relevant to Dot Dencity

$n$ Number of displayed dots in each of the first and second display tields Exposure duration of each field

IFI Interfield interval; duration between the offect of the first and the onset of the second dipplay fleid

d The horfzontal or vertical component of the dipplacement of spatial position in millimeters (or virual andle) of the difplaced dot between the firot and second display field

IQI Interquery interval; time between second dimplay field and the query circle

A Aros of display; the $8 \times 8 \mathrm{~cm}$ display area was reduced by multiplying the coordinates of each point by the fraction $f$

pro Protective barrier about each point each point was centered upon equare with linear dimensions, pro. within which no other point could intrude.

sep Minimil separation between queried dot and displaced dot, where sep $>$ pro 


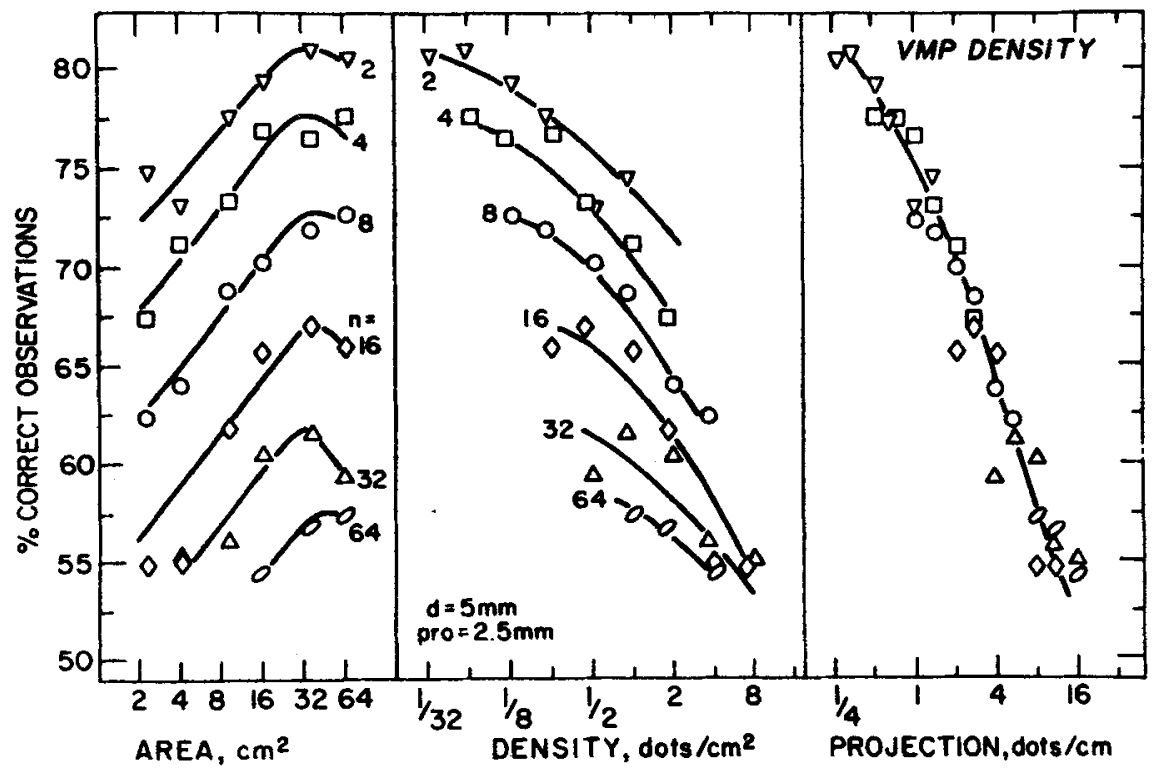

Fig. 1. Accuracy of identification of the states of the displacement of individually queried dots as a function of display area (left panel), of display density (middle panel), and of display projection (right panel). The parameter is the number of dots in the display. The displacement magnitude was $5 \mathrm{~mm}$; the magnitude of the protection feature was $2.5 \mathrm{~mm}$. right rotation of $n$ bits for $2^{-n}$ reduction and by combining rotations for reductions of $3 / 16,3 / 8$, and $3 / 4$.) The various display sizes overlapped in their upper right corners. 2

The protection feature, pro, placed an invisible square barrier about each point. Consider a $6 \times 6 \mathrm{~cm}$ display surface. With deliberate positioning, it is just possible to place 9 dots, each centered within a square protected region $2 \mathrm{~cm}$ on a side. However, if we first deliberately positioned the first dot at the dead center of the display, it is unlikely by a 10-bit random selection ( 2 chances in 1,024 ) to select a y-coordinate which satisfactorily positions another dot within the subscribed protection. Failures to generate a display of $n$ randomiy positioned dots were found when pro (in millimeters) $\leqslant 40 / \sqrt{n}$ for the full-sized display and $f($ pro $) \leqslant 40 / \sqrt{n}$ for the smaller displays. For this reason, the entire range of variables could not be examined over all display areas. If a given randomly selected position did not satisfy the protection feature with respect to a previously selected position, a new random condition of a single displaced dot. For, in order to detect whether or not there was any change in the display, $O$ would have to note the displacement of the single displaced dot. There is, however, an additional requirement imposed when a specific dot is queried. $O$ may have correctly noted the displacement, but, because the displaced dot was so close to a neighboring dot, it was not possible for $O$ to determine whether the queried dot was the displaced dot or simply a neighboring dot. This alternative will be referred to as the separation hypothesis.

\section{Experimental Variables}

The $O$ was seated $210 \mathrm{~cm}$ from the display. At this distance, a displacement of $5 \mathrm{~mm}$ is equivalent to a visual angle of about $8 \mathrm{~min}$. Natural binocular viewing was employed. Background illumination was dim. For the display duration, $t=91 \mathrm{msec}$; the interflash interval (IFI) $=91 \mathrm{msec}$; and the interquery interval (IQI) = 178 msec.

The last three parameters of Table 1 are of special interest to the present tests. The largest display was $8 \times 8 \mathrm{~cm}$ on the $8 \times 10 \mathrm{~cm}$ display surface. Smaller display areas were achieved by generating the patterns appropriate for the full display surface and then multiplying each coordinate by a fractional component, $f$, of the reduced display. Fractional components of $3 / 16,1 / 4,3 / 8,1 / 2$, $3 / 4$, and 1 were employed. (Operationally, this was achieved by

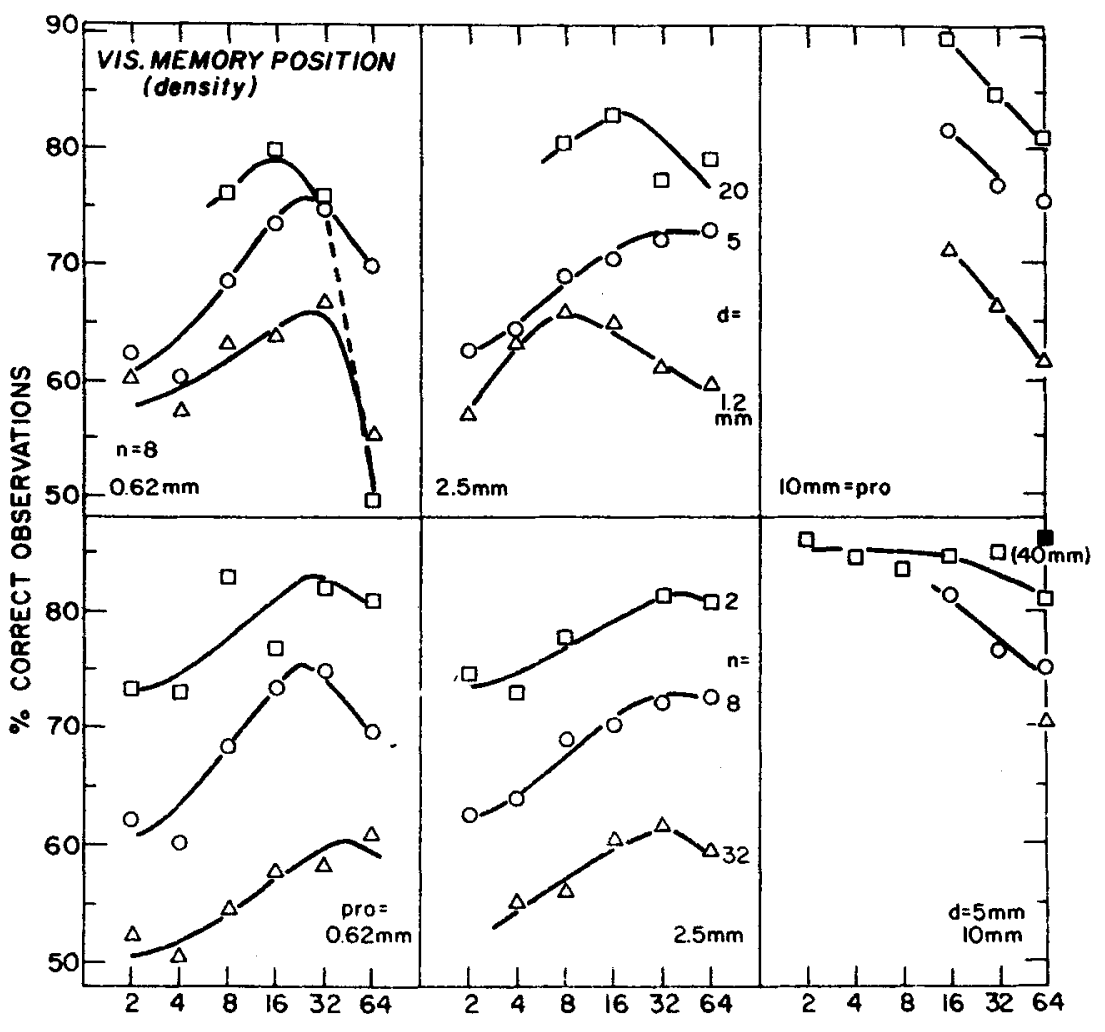

DISPLAY AREA, $\mathrm{cm}^{2}$

Fig. 2. Accuracy of identification of the state of displacement of individually queried dots as a function of display area. The left, middle, and right sections represent three protection levels: $0.62,2.5$, and $10 \mathrm{~mm}$, surrounding each dot. The parameter on the curves of the lower row is the number of dots in the display; the parameter on the curves of the upper row is the magnitude of displacement. Tests in the lower row employed a constant displacement of $5 \mathrm{~mm}$; tests in the upper row employed a constant number of dots, $n=8$. The filled point represents a protection of $40 \mathrm{~mm}$. 
selection was drawn. Note also that as pro approaches its limit, the dots within the display tend to be more evenly spaced than would be obtained by unconstrained random selection alone.

In addition to the protection feature, pro, which was applied to both experimental paradigms, a separation feature, sep, was applied in special identification tests of the state of a specific dot. A dot was generated at distance, sep, from the location of the single displaced dot within the second display field. Its direction was randomly chosen among the $\mathrm{NE}, \mathrm{SE}$, $\mathrm{SW}$, or NW directions. On one-half of the trials, the displaced dot was queried; on one-half of the trials, the dot separated by sep was queried. In these tests, sep $\geqslant$ pro.

Over the course of the experiment, 264 conditions sampled the experimental variables. Each $\mathrm{O}$ worked individually. Feedback was provided after each observation. Trials were blocked by 25 successive observations under a given set of parameters. Each condition is represented by about 32 blocks, contributed by 14-18 university students, for a total of about $.2 \times 10^{6}$ observations.

\section{RESULTS AND DISCUSSION}

\section{A. Specific Dot Queried}

We shall first examine tests in which the task was to identify whether the queried dot had, or had not, been displaced.

Figure 1 presents the results of tests in which the display was varied from $1.5 \times 1.5 \mathrm{~cm}$ to $8 \times 8 \mathrm{~cm}$ in, roughly, steps of area of $2: 1$ with a fixed protection region, $2.5 \mathrm{~mm}$, about each dot. In all tests, only a single dot was displaced between the first and second display fields. The abscissa of the left section is the display area, $A$; that of the middle section is the dot density, $\mathrm{n} / \mathrm{A}$; and that of the right section is related to a linear projection of dot density, $n / \sqrt{A}$. The parameter on the curves is the number of dots in the display, n. A set of nearly parallel performance curves is developed as a function of area (left section), of dot density (middle section), and of dot projection (right section) as a function of $n$. Performance improves with the area of display, at least to areas of $36 \mathrm{~cm}^{2}$. The fall in performance at the largest display area in Fig. 1 , and in subsequent figures, may be related to nonfoveal viewing or to the sharing of attention over display area, as initially mentioned. For a fixed display area, a change in performance of about $20 \%-25 \%$ correct observations is associated with the number of dots (left panel) under the conditions examined.

There clearly is an effect of dot

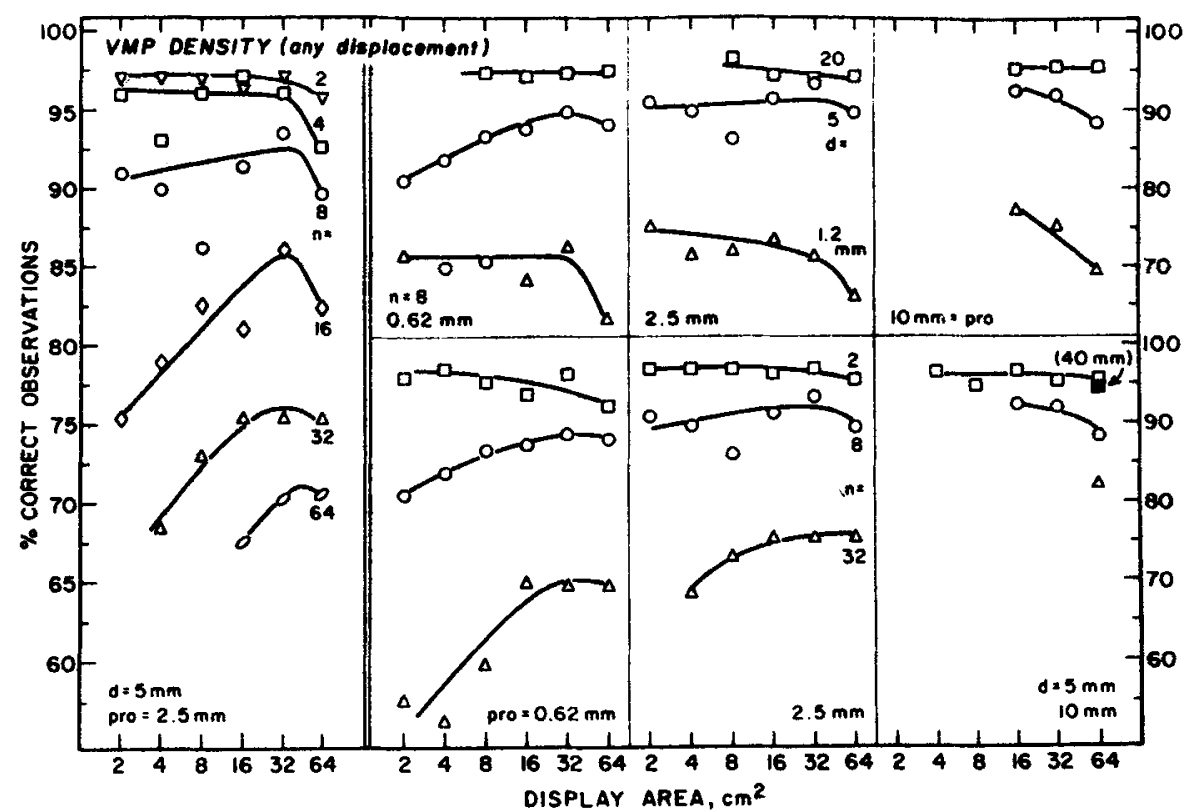

Fig. 3. Accuracy of identification of any displacement within a display as a function of display area. The organization of the left panel parallels the left panel of Fig. 1 ; the organization of the right panel parallels that of Fig. 2.

number which is independent of display density (middle panel). For a fixed dot density, a change in performance of about $10 \%-15 \%$ is associated with the number of dots-about half of that assignable to the number of dots as a function of display area. Over the rising portion of the curves within the first section, performance in the right panel is nearly invariant with $n$ for fixed levels of $n / \sqrt{A}$, the number of dots per linear display extent. This invariance is puzzling, since the separation of neighboring dots is directly related to dot density. The invariance with $n / \sqrt{\mathrm{A}}$ implies that discrimination is based upon the density of projection upon the component axes. This implication appears unreasonable.

Performance might suffer at higher dot density because dot displacement goes unnoted at the higher display densities or because the displacement is noted, but $O$ cannot discriminate the queried dot from its immediate neighbors on the display. The latter alternative will be called the separation hypothesis. There are several ways to distinguish between these alternatives.

Figure 2 considers tests in which the magnitude of the protection feature-the safety region about each point-was varied. We shall tentatively assume that larger protection regions about each point serve more to reduce confusions between the queried dot and its neighbors than to aid the detection of displacement. This proposition will be tested later more directly.
The middle panel of the bottom row of Fig. 2 replots three of the curves of the left section of Fig. 1. The parameter is the number of dots in the display. With a fourfold reduction (left panel of bottom row relative to the middle panel) in the linear protection feature-a 16-fold reduction in the protected area-there is little change in discriminability, except at $n=32$ with a $4 \%$ average loss in performance. With a fourfold increment in the linear protection feature (right panel of bottom row relative to the middle panel), however, there is a sharp gain in performance. With $n=2$, the only condition spanning the entire range of areas, performance is relatively independent of display area at the highest protection level. If anything, performance is somewhat better with the smaller displays. Only a restricted range of display areas met the requirement of a fixed protection distance with $n=8$ and $n=32$. With $\mathbf{n}=8$, performance also fails to improve with the larger displays. Overall, there is an average $7 \%$ gain in performance relative to the corresponding conditions of the middle panel. These results suggest that losses of performance noted with analler displays may be tied to the protection feature. If pro is sufficiently large, performance does not suffer with smaller displays. In fact, with a large protected region about each point, higher scores are obtained with the smaller displays.

The protection feature might assist discrimination by spreading out all 


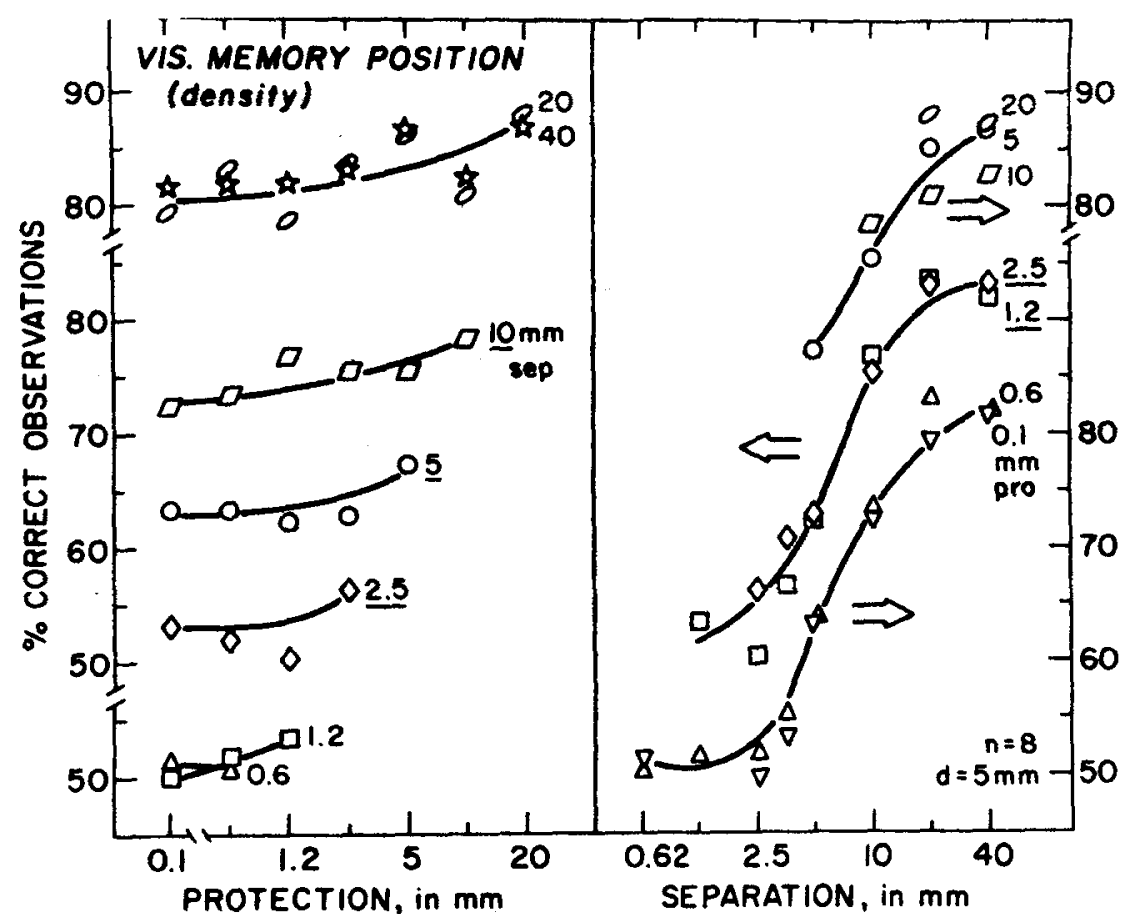

Fig. 4. Accuracy of identification of the state of displacement of individually queried dots as a function of the magnitude of the protection feature (left panel) and of the separation feature (right panel). Successive curves are displaced in the left panel as a function of sep: $0.6,1.2 \mathrm{~mm} ; 2.5,5,10 \mathrm{~mm}$; and $20,40 \mathrm{~mm}$; successive curves are displaced in the right panel as a function of pro: 0.1 , $0.6 \mathrm{~mm} ; 1.2,2.5 \mathrm{~mm}$; and 5, 10, $20 \mathrm{~mm}$. The scores for the bottom set of points (lowest sep and pro levels) are scaled on the right ordinate.

dots and/or, in particular, by spacing out the distance between the queried dot and its neighbors.

The latter alternative is supported by the results of the upper row of Fig. 2. The extent of displacement, plotted as the parameter on the curves, was varied. It is tentatively assumed that the detection of displacement is primarily related to the magnitude of displacement rather than to the separation between the queried dot and neighbors. The left, middle, and right panels consider the same protected regions represented on the lower row. Overall, performance improves with larger dot displacements. Yet, the previously noted result of Fig. 2 remains. With large protection (right panel of upper row), performance again falls with display area over a range of displacements. Most striking is the plunge in performance (dashed line in the left panel of upper row) associated with the largest displacement, largest area, and smallest protection-a condition favorable to the detection of displacement but unfavorable to the isolation of the queried dot from its neighbors.

B. Any Display Displacement

We shall next examine tests which closely parallel those of the first section. On $50 \%$ of the observations, a single dot was displaced; on $50 \%$ of the observations, no dot was displaced. The task was to identify whether any dot had or had not been displaced.

The left panel of Fig. 3 asks how the detection of any displacement is related to the number of dots in the display (parameter) and to the display area (abscissa). The conditions directly parallel those of Fig. 1. Comparison between the left panels of Figs. 1 and 3 reveals substantially higher performance in identifying whether any displacement has taken place relative to identifying whether or not a specific dot has been displaced. Since only a single dot was displaced in each case, the differences in performance are presumably more related to the accuracy of separating the queried dot from its neighbors than to the detection of any displacement. The consistent gain in performance with display area previously noted in Fig. 1 is obtained in Fig. 3 only for a large number of dots in the display. For displays of 8 dots or less, performance changes little with display area. The slight downturn noted in Fig. 1 for the largest display is also observed in Fig. 3 in identifying any displacement. The six panels of the right section of Fig. 3 and the right ordinate parallel the conditions of Fig. 2. Again, we note the lesser dependence upon display area in identifying any displacement and poorer performance at the larger display areas with the larger protection regions.

\section{The Separation Hypothesis}

The decrement in performance with dot density noted in Fig. 1 might result because more dense packing reduces the detection of any displacement or because more dense packing reduces the distance between the queried dot and its immediate neighbors.

Partial support was provided for each of the alternatives. Figure 3 showed that detection of any displacement suffers at smaller display areas, at least for $n>16$. Moreover, the detection of any displacement also improves with the protection feature, pro, which serves to spread out the dots. Since the relative magnitude of the various effects tend to be less for detection of any displacement than for the state of a specific dot, indirect evidence was also furnished for the second alternative.

More direct evidence for the second alternative is furnished by tests in which the protection and separation features were independently varied (within previously noted constraints). As noted under Method, the protection feature simply imposes a lower limit upon random selection; the sep feature places a nondisplaced dot exactly at distance sep from the position of the displaced dot in the second display field.

Figure 4 considers the effect of sep and pro. The abscissa of the left section is the magnitude of the protection feature, pro, and the parameter is the magnitude of the separation feature, sep. Successive curves are displaced vertically to avoid overlapping, as noted in the figure legend. The abscissa and parameter are exchanged in the right section.

For a fixed separation between the displaced dot and a neighboring dot, there is only a small effect of the protection feature (left section). For a fixed protection among all dots, there is a large effect of the separation feature (right section). The results suggest that the strong effect of the protection feature in Fig. 2 was primarily manifested through the indirect relation, sep $>$ pro.

It is noted that Fig. 4 considers the proportion of correct observations, averaged over queries for both displaced and nondisplaced dots. In the case of a single displaced dot, $O$ reports he monitors the display for the single displaced dot rather than for the other nondisplaced dots. We might therefore anticipate a response bias in 
favor of the response "displaced" when uncertain. Indeed, at sep levels of $\leqslant 2.5 \mathrm{~mm}$, there is a $14 \%$ correct advantage in favor of querying displaced, relative to nondisplaced, dots. Moreover, the sep feature specifies the distance between the displaced dot and the query dot only on that subset of trials in which the nondisplaced dot is queried. Relative to scores when the displaced dot is queried, scores when the nondisplaced dot is queried are smaller for smaller sep levels and are larger for larger sep levels. For sep levels of $\leqslant 2.5,5$, $10 \mathrm{~mm}$ and $\geqslant 20 \mathrm{~mm}$, the advantage when querying the nondisplaced dot is $-14 \%,-3 \%, 6 \%$, and $9 \%$ correct, respectively, relative to querying the displaced dot. Stated differently, the effect of the separation variable is even larger than that shown in Fig. 4 when restricted to the subset of observations in which the nondisplaced dot is queried.

Finally, Fig. 5 shows that the role of the separation feature is predominant to the protection feature over a wide range of display areas. It is also noted that display area-and thereby display density - still plays a small role even when the protection and separation features of the displays are held constant.

\section{SUMMARY}

For a single dot displaced between the successive random dot fields, detection of any displacement is substantially better than identification of the state of a specifically queried dot. If we assume that the accuracy of detection of any displacement is equivalent in both tasks and since only

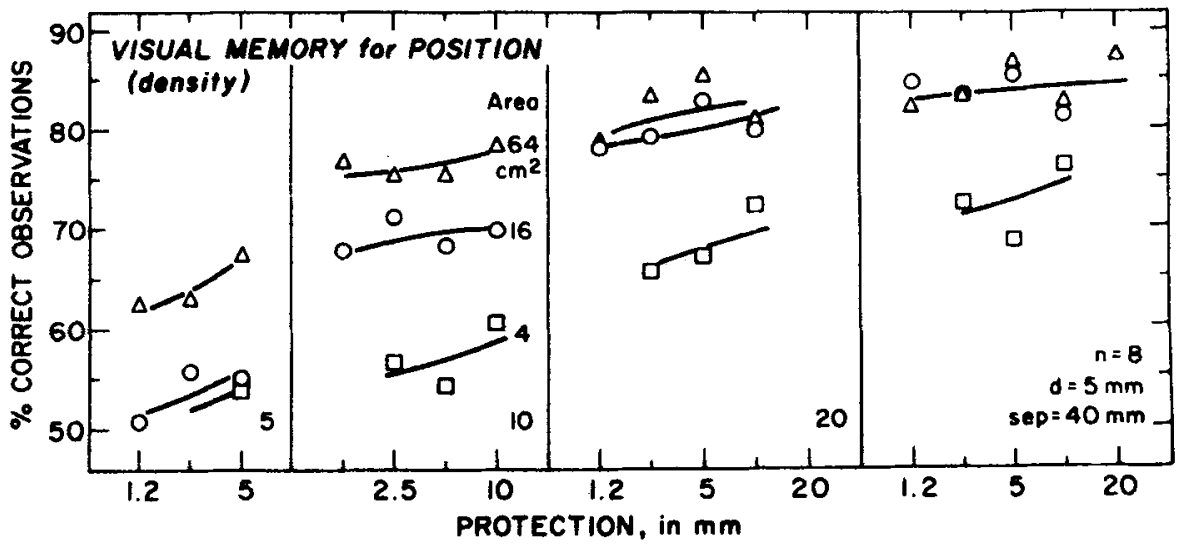

Fig. 5. Accuracy of identification of the state of displacement of individually queried dots as a function of the magnitude of the protection feature (abscissa), of the separation feature (separate panels), and of display area (parameter).

one dot was displaced, it seems reasonable that the poorer performance of the second task is related to factors associated with the identification of the queried dot. In particular, the separation between the displaced dot and its immediate neighbors may be implicated. Indirect evidence for the presumption was furnished by the role of display density and by a "protection" feature separating all of the points of the display. Direct evidence was furnished by control of the separation of the displaced dot and of the queried nondisplaced dot.

\section{REFERENCES}

ERIKSEN, C. W., \& ROHRBAUGH, J. W Some factors determining efficiency of selective attention. American Journal of Psychology, 1970, 33, 330-342.
POLLACK, I. Detection of changes in spatial position: Short-term visual memory or motion perception? Perception \& Psychophysics, 1972, 11 17-27.

RUMELHART, D. A multicomponent theory of the perception of briefly exposed visual displays. Journal of Mathematical Psychology, 1970, 7 , 191-218.

SEKULER, R. W., \& ABRAMS, M. Visual sameness: $A$ choice time analysis of pattern recognition processes. Journal of Experimental Psychology, 1968, 77, 232-238.

\section{NOTES}

1. Pollack. I Detection of changes in spatial position: II. Delayed recognition of individual elements. In preparation.

2. An alternative method for dissociating number of elements and subtended area has been provided by Eriksen and Rohrbaugh (1970).

(Received for publication July 31, 1972.) 